\title{
Barriers and facilitators of reporting foodborne illness
}

\author{
Jonathan L. Chua* and Mark Chen \\ National University of Singapore, Singapore, Singapore
}

\section{Objective}

To better understand the barriers and facilitators to reporting and assessing what improvements would increase participation.

\section{Introduction}

Traditional surveillance methods have a major challenge to estimating the burden of disease due to underreporting [1]. Participatory surveillance techniques can help supplement to monitor and detect foodborne outbreaks while reducing the impact of underreporting [2]. As there is a low participation rate in Singapore, this study aims to better understand the barriers and facilitators to reporting and assesses what improvements can increase participation.

\section{Methods}

A total of 14 individuals participated in the study; 8 had informed health authorities of a possible foodborne outbreak while 6 patients were diagnosed with gastroenteritis at general practitioner (GP) clinics but did not report their illness to health authorities. We examined the barriers and facilitators to reporting foodborne illnesses to health authorities through semi-structured in-depth interviews and thematic analysis.

\section{Results}

The median age of participants was 28 (Interquartile Range = 23-37). The majority were Singaporeans and had or were pursuing university qualifications. The combination of perceived severity of illness and degree of certainty of the cause of illness are key reasons that are both barrier and facilitator to reporting foodborne illness to the authorities. The informants expected government intervention and hoped that their actions would prevent others from being affected as well. However, reporting to health authorities was usually delayed by the participants' severity of illness. Those who did not report were unaware of reporting channels and were concerned their actions would negatively affect food establishments.

Participants also shared what they would like to see in a reporting system. Firstly, contact information should be easily accessible with a user-friendly system. Secondly, a human touch and live acknowledgement was desirable when reporting the issue instead of being met with recorded voice machine messages. This would also reduce the number of subsequent follow up calls from the authorities to gather information.

\section{Conclusions}

It is crucial for the public to be informed of easily accessible and user-friendly avenues to report foodborne incidences to the authorities. Being able to communicate directly to relevant authorities immediately would help relay issues with the assurance that the matter would be looked into and acted upon. In trying to understand the barriers and facilitators to reporting, the study hopes to see a higher motivation of the public to report, so that necessary actions can be taken to reduce foodborne incidences.

\section{Keywords}

Participatory surveillance; Underreporting; Public health surveillance; Barriers and facilitators

\section{Acknowledgments}

We would like to acknowledge the Foodborne outbreak team within the Communicable Disease Division of the Ministry of Health, Singapore for helping to recruit participants. Also we would like to thank Frontier Medical and University Health Centre for allowing us to recruit participants at their clinics. Lastly, we acknowledge the funding from National Medical Research Council, Singapore.

\section{References}

1. Fletcher SM, Lewis-Fuller E, Williams H, et al. Magnitude, distribution, and estimated level of underreporting of acute gastroenteritis in Jamaica. J Heal Popul Nutr. 2013;31(4 SUPPL.1):69-80. http://www. ncbi.nlm.nih.gov/pubmed/24992813. Accessed October 4, 2017.

2. Quade P, Nsoesie EO. A Platform for Crowdsourced Foodborne Illness Surveillance: Description of Users and Reports. JMIR Public Heal Surveill. 2017;3(3):e42. doi:10.2196/publichealth.7076.

\section{*Jonathan L. Chua}

E-mail: jonathanlumenchua@gmail.com 